Yan Xiao and Chenzhong Cao*

\title{
Crystal structure of bis\{5-chloro-2-(((4- trifluoromethyl)imino)methyl)phenolato- $\left.\mathrm{k}^{2} \mathrm{~N}, \mathrm{O}\right\}$ copper(II), $\mathrm{C}_{28} \mathrm{H}_{16} \mathrm{Cl}_{2} \mathrm{CuF}_{6} \mathrm{~N}_{2} \mathrm{O}_{2}$
}

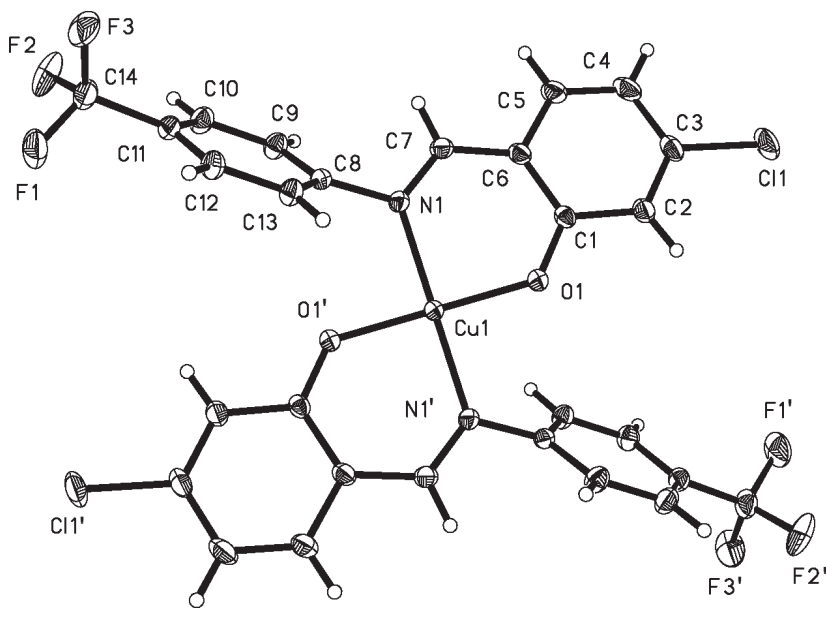

https://doi.org/10.1515/ncrs-2020-0162

Received March 26, 2020; accepted April 18, 2020; available online May 5, 2020

\section{Abstract \\ $\mathrm{C}_{28} \mathrm{H}_{16} \mathrm{Cl}_{2} \mathrm{CuF}_{6} \mathrm{~N}_{2} \mathrm{O}_{2}$, monoclinic, $P 2_{1} / c \quad$ (no. 14),

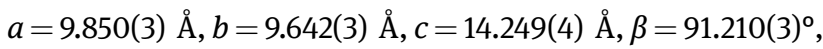 $V=1352.9(6) \AA^{3}, Z=2, \quad R_{\mathrm{gt}}(F)=0.0377, \quad w R_{\text {ref }}\left(F^{2}\right)=0.1066$, $T=296 \mathrm{~K}$.}

\section{CCDC no.: 1992337}

The molecular structure is shown in the figure. Table 1 contains crystallographic data and Table 2 contains the list of the atoms including atomic coordinates and displacement parameters.

*Corresponding author: Chenzhong Cao, School of Resource Environment and Safety Engineering, School of Chemistry and Chemical Engineering, Key Laboratory of Theoretical Organic Chemistry and Function Molecule, Ministry of Education, Hunan University of Science and Technology, Xiangtan 411201, P.R. China, e-mail:czcao@hnust.edu.cn

Yan Xiao: School of Resource Environment and Safety Engineering, School of Chemistry and Chemical Engineering, Key Laboratory of Theoretical Organic Chemistry and Function Molecule, Ministry of Education, Hunan University of Science and Technology, Xiangtan 411201, P.R. China. https://orcid.org/0000-0001-6404-9725

๑ Open Access. ( 2020 Yan Xiao et al., published by De Gruyter. (c) BY License.

Table 1: Data collection and handling.

\begin{tabular}{ll}
\hline Crystal: & Brown block \\
Size: & $0.28 \times 0.24 \times 0.16 \mathrm{~mm}$ \\
Wavelength: & Mo $K \alpha$ radiation $(0.71073 \AA)$ \\
$\mu:$ & $1.08 \mathrm{~mm}^{-1}$ \\
Diffractometer, scan mode: & Bruker APEX-II, $\varphi$ and $\omega$ \\
$\theta_{\text {max }}$, completeness: & $26.0^{\circ},>99 \%$ \\
$N(h k l)_{\text {measured }}, N(h k l)_{\text {unique }}, R_{\text {int }}:$ & $9409,2643,0.030$ \\
Criterion for $I_{\text {obs }}, N(h k l)_{\text {gt }}:$ & $I_{\text {obs }}>2 \sigma\left(I_{\text {obs }}\right), 2127$ \\
$N(\text { param })_{\text {refined }}:$ & 187 \\
Programs: & Bruker [1], SHELX [2], Olex2 [3] \\
\hline
\end{tabular}

Table 2: Fractional atomic coordinates and isotropic or equivalent isotropic displacement parameters $\left(\AA^{2}\right)$.

\begin{tabular}{lrrrr}
\hline Atom & $\boldsymbol{x}$ & $\boldsymbol{y}$ & $\boldsymbol{z}$ & $\boldsymbol{U}_{\text {iso }}{ }^{*} \boldsymbol{U}_{\text {eq }}$ \\
\hline Cu1 & 0.500000 & 0.500000 & 0.500000 & $0.03283(16)$ \\
Cl1 & $0.02308(9)$ & $0.61595(13)$ & $0.83207(6)$ & $0.0838(4)$ \\
F1 & $0.4436(3)$ & $0.3164(3)$ & $-0.00093(13)$ & $0.0897(7)$ \\
F2 & $0.2325(2)$ & $0.3016(3)$ & $0.00824(15)$ & $0.1068(10)$ \\
F3 & $0.3257(3)$ & $0.4932(3)$ & $-0.03235(14)$ & $0.1034(10)$ \\
O1 & $0.39007(19)$ & $0.4953(2)$ & $0.60698(12)$ & $0.0423(5)$ \\
N1 & $0.3386(2)$ & $0.5246(2)$ & $0.41516(14)$ & $0.0354(5)$ \\
C1 & $0.2689(3)$ & $0.5461(3)$ & $0.61597(17)$ & $0.0367(6)$ \\
C2 & $0.2123(3)$ & $0.5512(3)$ & $0.70646(19)$ & $0.0457(7)$ \\
H2 & 0.260425 & 0.514686 & 0.757612 & $0.055^{\star}$ \\
C3 & $0.0880(3)$ & $0.6092(3)$ & $0.7190(2)$ & $0.0519(8)$ \\
C4 & $0.0099(3)$ & $0.6646(4)$ & $0.6450(2)$ & $0.0603(9)$ \\
H4 & -0.073347 & 0.706685 & 0.655610 & $0.072^{*}$ \\
C5 & $0.0599(3)$ & $0.6549(3)$ & $0.5573(2)$ & $0.0526(8)$ \\
H5 & 0.007885 & 0.688513 & 0.507054 & $0.063^{*}$ \\
C6 & $0.1880(3)$ & $0.5958(3)$ & $0.53931(18)$ & $0.0399(6)$ \\
C7 & $0.2249(3)$ & $0.5756(3)$ & $0.44409(18)$ & $0.0400(6)$ \\
H7 & 0.161282 & 0.601413 & 0.398191 & $0.048^{*}$ \\
C8 & $0.3447(3)$ & $0.4960(3)$ & $0.31592(17)$ & $0.0353(6)$ \\
C9 & $0.3175(3)$ & $0.5966(3)$ & $0.24975(19)$ & $0.0469(7)$ \\
H9 & 0.300120 & 0.687123 & 0.268453 & $0.056^{*}$ \\
C10 & $0.3161(3)$ & $0.5629(4)$ & $0.1550(2)$ & $0.0510(7)$ \\
H10 & 0.296750 & 0.630692 & 0.110272 & $0.061^{*}$ \\
C11 & $0.3434(3)$ & $0.4294(3)$ & $0.12746(17)$ & $0.0436(7)$ \\
C12 & $0.3755(3)$ & $0.3293(3)$ & $0.19340(19)$ & $0.0462(7)$ \\
H12 & 0.395688 & 0.239455 & 0.174387 & $0.055^{*}$ \\
C13 & $0.3777(3)$ & $0.3626(3)$ & $0.28777(18)$ & $0.0426(6)$ \\
H13 & 0.401240 & 0.295780 & 0.332294 & $0.051^{*}$ \\
C14 & $0.3346(4)$ & $0.3876(4)$ & $0.0264(2)$ & $0.0629(9)$ \\
\hline & & & &
\end{tabular}
This work is licensed under the Creative Commons Attribution 4.0 International 


\section{Source of material}

The Schiff base ligand 5-chloro-2-(((4-(trifluoromethyl)imino) methyl)phenol was prepared according to the literature method [4]. The copper(II) acetate monohydrate (10 mmol) dissolved in ethanol solution $(10 \mathrm{~mL})$ was added dropwise to the ligand $(20 \mathrm{mmol})$ dissolved in ethanol $(10 \mathrm{~mL})$ at room temperature. The mixture was kept being stirred for $1 \mathrm{~h}$. The filtrate was allowed to stand for 35 days at quiet environment. Several brown crystal were obtained.

\section{Experimental details}

$\mathrm{H}$ atoms linked to $\mathrm{N}, \mathrm{C}$ and $\mathrm{O}$ atoms were geometrically added and isotropically refined by the riding model.

\section{Comment}

Schiff bases ligands have been attracting much attention because of their excellent coordination ability to metal ions $[5,6]$, and their complexes exhibited magnetic properties and many other ones [7]. So far, we have synthesized a series of bidentate Schiff base copper complexes [8]. Particular attention has been paid to the structural and spectroscopic properties of Schiff base metal complexes $[9,10]$.

In the title crystal structure, the coordination sphere of $\mathrm{Cu}$ atom is found to be formed by two bidentate ligands. Each $\mathrm{Cu}$ atom in the complex is four-coordinated by two imino $\mathrm{N}$ and two phenolato $\mathrm{O}$ atoms of the corresponding Schiff base ligand, forming a square-planar coordination. The Cu1-N1 bond lengths are both 1.991(2), and the Cu1-O1 bond lengths are both 1.8889(18). The angles of N1-Cu1-O1 and N1-Cu1-O1' are 88.35(9) and 91.65(9), respectively.

Acknowledgements: The work was supported by the National Natural Science Foundation of China (21672058).

\section{References}

1. Bruker. APEX2, SAINT and SADABS. Bruker AXS Inc., Madison, WI, USA (2012).

2. Sheldrick, G. M.: Crystal structure refinement with SHELXL. Acta Crystallogr. C71 (2015) 3-8.

3. Dolomanov, O. V.; Bourhis, L. J.; Gildea, R. J.; Howard, J. A. K.; Puschmann, H.: OLEX2: A complete structure solution, refinement and analysis program. J. Appl. Crystallogr. 42 (2009) 339-341.

4. Zhao, J. X.; Wu, Q.: Synthesis and crystal structure of bis\{5-fluorine-2-(((4-(1-(methoxy-imino)ethyl)phenyl) imino)methyl)phenolato- $\mathrm{K}^{2} \mathrm{~N}, \mathrm{O}$ \}copper(II), $\mathrm{C}_{32} \mathrm{H}_{28} \mathrm{CuF}_{2} \mathrm{~N}_{4} \mathrm{O}_{4}$. Z. Kristallogr. NCS 235 (2020) 257-258.

5. Zhao, L.; An, Q. Q.; Zhao, J. X.; Li, P. P.; Wang, F.: Crystal structure of bis(2-((E)-((4-((E)-1-(ethoxyimino)ethyl)phenyl)imino) methyl)-5-methoxyphenolato- $\left.\mathrm{K}^{2} \mathrm{O}, \mathrm{N}\right)$ copper(II), $\mathrm{C}_{36} \mathrm{H}_{38} \mathrm{CuN}_{4} \mathrm{O}_{6}$. Z. Kristallogr. NCS 232 (2017) 595-597.

6. Mohapatra, R. K.; Sarangi, A. K.; Azam, M.; El-ajaily, M. M.; Kudrat-E-Zahan, M.; Patjoshi, S. B.; Dash, D. C.: Synthesis, structural investigations, DFT, molecular docking and antifungal studies of transition metal complexes with benzothiazole based Schiff base ligands. J. Mol. Struct. 1179 (2019) 65-75.

7. Jana, S.; Shaw, B. K.; Bhowmik, P.; Harms, K.; Drew, M. G. B.: Field-induced ferromagnetism and multiferroic behavior in end-on pseudohalide-bridged dinuclear copper(II) complexes with tridentate schiff base blocking ligands. Inorg. Chem. 53 (2014) 8723-8734.

8. Xiao, Y.; Cao, C.: Influence of substituents on the structure of Schiff bases Cu(II) complexes. J. Mol. Struct. 1209 (2020) 1-6.

9. Li, P. P.; Zhao, L.; Zhao, J. X.; Zhu, Z. B.; Wang, F.; An, Q. Q.: Synthesis and crystal structure of bis $\{1-(((4-(1-$ (hydroxyimino)ethyl)phenyl)imino)methyl)naphthalen-2-olato$\mathrm{K}^{2} \mathrm{O}, \mathrm{N}$ \}copper(II), $\mathrm{C}_{38} \mathrm{H}_{30} \mathrm{CuN}_{4} \mathrm{O}_{4}$. Z. Kristallogr. NCS 232 (2017) 889-890.

10. Xiao, Y.; Cao, C.: Influence of substituent effects on the coordination ability of salicylaldehyde Schiff bases. J. Coord. Chem. 71 (2018) 3836-3846. 